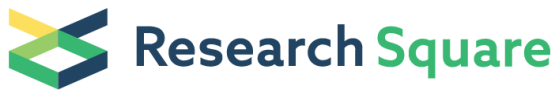 \\ Preprints are preliminary reports that have not undergone peer review. \\ They should not be considered conclusive, used to inform clinical practice, or referenced by the media as validated information.
}

\section{Identification of pulmonary comorbid diseases network based repurposing effective drugs for COVID-19}

\section{Jai Chand Patel}

Defence Institute of Physiology and Allied Sciences, Defence Research and Development Organization

\section{Rajkumar Tulswani}

Defence Institute of Physiology and Allied Sciences, Defence Research and Development Organization

\section{Pankaj Khurana}

Defence Institute of Physiology and Allied Sciences, Defence Research and Development Organization

\section{Yogendra Kumar Sharma}

Defence Institute of Physiology and Allied Sciences, Defence Research and Development Organization

\section{Lilly Ganju}

Defence Institute of Physiology and Allied Sciences, Defence Research and Development Organization

\section{Bhuvnesh Kumar}

Defence Institute of Physiology and Allied Sciences, Defence Research and Development Organization

\section{Ragumani Sugadev ( $\nabla$ ragusugadev@gmail.com )}

Defence Institute of Physiology and Allied Sciences, Defence Research and Development Organization

\section{Research Article}

Keywords: COVID-19, comorbid diseases, drug repurposing, bioinformatics, rat study, resveratrol

Posted Date: May 11th, 2020

DOI: https://doi.org/10.21203/rs.3.rs-28148/v1

License: (c) (i) This work is licensed under a Creative Commons Attribution 4.0 International License. Read Full License 


\section{Abstract}

The number of hospitalization of COVID-19 patients with one or more comorbid diseases is highly alarming. Despite the lack of large clinical data and incomplete understanding of virus pathology, identification of the COVID-19 associated diseases with clinical precision are highly limited. In this regard, our text mining of 6238 PubMed abstracts (as on 23 April 2020) successfully identified broad spectrum of COVID-19 comorbid diseases/disorders (54), and their prevalence on the basis of the number of occurrence of disease terms in the abstracts. The disease ontology based semantic similarity network analysis revealed the six highly comorbid diseases of COVID-19 namely Viral Pneumonia, Pulmonary Fibrosis, Pulmonary Edema, Acute Respiratory Distress Syndrome (ARDS), Chronic Obstructive Pulmonary Disease (COPD) and Asthma. The disease gene bipartite network revealed 15 genes that were strongly associated with several viral pathways including the corona viruses may involve in the manifestation (mild to critical) of COVID-19. Our tripartite network- based repurposing of the approved drugs in the world market revealed six promising drugs namely resveratrol, dexamethasone, acetyl cysteine, Tretinoin, simvastatin and aspirin to treat comorbid symptoms of COVID-19 patients. Our animal studies in rats and literatures strongly supported that resveratrol is the most promising drug to possibly reduce several comorbid symptoms associated with COVID-19 including the severe hypoxemia induced vascular leakage. Overall, the anti-viral properties of resveratrol against corona virus could be readily exploited to effectively control the viral load at early stage of COVID-19 infection through nasal administration.

\section{Introduction}

The coronavirus 2019 (COVID-19) positive cases across the world crossed 2544792 and the death toll topped 175694 as on $23^{\text {rd }}$, April 2020, since its outbreak in early December 1 . The number of recovered cases from coronavirus, too, has increased to 700,000 . Almost all the countries in the world are still struggling to keep the virus in control and facing huge economic recession due to lockdown $\underline{2}$. Now the entire world is in desperate need for drugs and vaccines to save our mankind from the pandemic. In this scenario, researchers from all over the world working together to develop drugs to control in its four stages of disease progression.

The early (first) stage of the disease control starts with controlling at the zero-point entry. The virus is primarily spread between people during close contact, often via small droplets sometimes ultrafine mist produced by coughing, sneezing, or talking $\stackrel{3}{ }$. Prevention is always better than cure. As per WHO guidance the most common preventive measures include hand washing, covering mouth while coughing, social distancing and self-isolation for people who are suspect to be infected ${ }^{4}$. But these guidelines are not full proof and does not guarantee the complete protection against the deadly virus. Obviously, debates are already started in the scientific community about the air borne transmission of COVID-19 5 . Once it is spread from the infected persons, major entry points point of the virus are thin epithelial layers of noise and throat, and the viral loads are highest in the nose than the throat $\underline{6}, \underline{z}$. The early symptoms onset starts 
since the day of infection varied from 7 to 32 days with a maximum viral load ${ }^{8}$. Most of the infected persons were cured with mild or no symptoms of sneezing, vomiting, fever and headache. But keep control of the viral load in these persons are much more crucial than the infected persons because they are more contiguous in spreading the virus $\underline{\underline{9}}$. There are numerous viruses follow the upper respiratory path for infection including the common cold influenzas virus $10,11,12,13$. Clinical studies supported that simple hypertonic saline nasal irrigation and gargling an ancient Yogic practice called "Jalaneti" significantly reduced the viral load in the influenza virus patients $\underline{14}, \underline{15}$. Keep in light, identifying an effective antiviral to reduce the viral load in the upper respiratory tract (say a nasal spray) could possibly be one of the best preventive measure to control COVID-19 pandemic.

The remaining three stages (mild, severe and critical) were classified according to the clinical manifestation of the diseases by their severity $\frac{16}{}$. Specific drugs or vaccines yet to arrive in the market, the most recommended drugs for COVID-19 patients are mainly used to control viral replication such as remdesivir, lopinavir/ritonavir, chloroquine and hydroxychloroquine blocking the viral proteins but with limited success rates $17,18,19, \underline{20}$. The most common mild and severe symptoms are highly related to the symptoms of pneumonia 21 . Many drugs are used to control the mild and severe symptoms of pneumonia of COVID-19 patients $\underline{22}$. Only $30 \%$ of severe population recovered, but others deteriorate, often quite suddenly, developing a condition called acute respiratory distress syndrome (ARDS) $\underline{23}, \underline{24}$. Importantly, the same diseases (pneumonia and ARDS) actually presents itself with impressive non-uniformity and distinctive features of severe hypoxemia often associated with near normal respiratory system compliance $\frac{25}{}$. Commonly, these patients end up on ventilators and many die. The autopsies of

the lung alveoli of these patients are filled with fluid, white blood cells, mucus, and the detritus of destroyed lung cells $\underline{26}$. These distinct etiology of COVID-19 necessitate the need to identify new drugs to treat the different features present in the three stages of severities.

The hospitalization rate for COVID-19 is 4.6 per 100,000 population, and almost $90 \%$ of hospitalized patients have one or more comorbidity $\underline{27}$. The number of patients admitted in the hospital shown severity in the diseases are having one or more comorbid diseases $\underline{28}$. Understanding the various comorbid diseases of COVID-19 could help the doctors in the front line to decide about the mode treatment at the earliest. For instance, hypertension, diabetes, and cardiovascular diseases are seeming to be the most common comorbidities in patients with COVID-19료,299. These diseases generally treated with reninangiotensin system (RAS), including angiotensin-converting enzyme inhibitors (ACEls) or angiotensin receptor blockers (ARBs) $\underline{30}, \underline{31}, \underline{32}$. Use of these ACEls found to be increased ACE2 levels in human intestine luminal cells and the ACE2 expression highly correlated with COVID-19 virulence ${ }^{33}$. Lei Fang et al., 
questioned the use of ACEls to treat COVID-19 patient $₫$ s severity $\underline{34}$. In this context, understanding different comorbidities of COVID-19 is essential for the frontline workers to decide about the mode of treatment strategies in advance.

Keep all the above in view, the current study had the following research objectives: (i) to collect all possible comorbid diseases of COVID-19 from literature mining especially from Pubmed (ii) to construct disease ontology based semantic similarity network to find the most likely comorbid diseases of COVID19 (iii) to further evaluate the disease comorbidity using gene - disease association network and understand the pathobiology of COVID-19 (iv) exploit the network to repurposing drugs to control the severity of COVID-19 (v) to experimentally verify the efficacy of the proposed drug to control severe hypoxemia (acute hypoxia) induced vascular leakage.

The main outcome of the analysis includes: (i) the disease ontology based semantic network revealed that highly comorbid diseases of COVID-19 were spread across many vital organs especially kidney besides lung and cardiac (ii) the bipartite network (Disease-gene network) revealed many respiratory disorder genes in the host cells were effectively utilized by several

viruses for their entry as well as replication (iii) tripartite network (disease-gene-drug network) revealed a

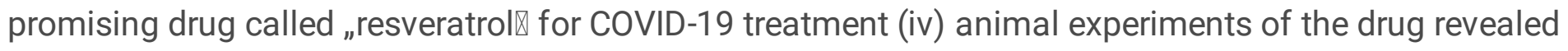
that the drug protect the rat lung from severe hypoxemia (acute hypoxia) induced vascular leakage (v) possible pros and cons of resveratrol to treat COVID-19 patients were discussed in detail.

\section{Results}

\section{Text mining based COVID-19 comorbid diseases Network}

The total number of unique disease terms associated with COVID-19 was 559. To reduce the influence of the noise on the network, for each disease, we analysed the number of occurance of disease terms (freqencies) from the 6238 abstracts (Supplementary Table 1). The wordcloud analysis revealed 54 diseases with their term frequencies range from 2 to 552 correspond to font size 1\% and 100\% (50 out of 54) respectively (Fig. 1, Supplementary Table 2). The strong disease associations were further ensured by DO score reduced the number of diseases to 54 (Supplementary Table 3). Finally, 44 diseases indicated a dense association among themselves (89 edges) with the DO score threshold at 0.5 (Fig. 2). Like many empirically observed biological networks, the disease-disease network was also a scale-free network whose degree distribution followed a power law (data not shown), that is most nodes connect to only a few other nodes whereas a few nodes act a hubs with a large number of links. The most connected 
disease hubs were clustered into five catogories (cluster 1 to 5 ) based on their topological properties of the network (Fig. 3 (A, B) Supplemantry Table 4). The cluster 1, 2, 3 and 4 consituted most densly connected networks with almost 10 diseases each. The cluster 1 was having the highest number of diseases with the node sizes $>10$ in 7 out of 44 diseases). In which earlier studies indicated that densly connected diseases in the network should shared similar Medical Subject Headings (MeSH) classsifications $\frac{35}{}$ (Fig. 3C). The DO terms of the core network were mapped to the MeSH communities and investigated whether they fell under similar disease MeSH classifications. For instance, Cluster 1 shared plumonary diseases were in the same classes of disease unique code with two sets of diseases closely defined in 3 digits. The set 1 diseases were Pulmonary edema (UID:D011654), Viral pneumonia (UID:D011024) and Pulmonary fibrosis (UID:D011658) whereas the set 2 diseaes were Asthma (UID:D001249) and Bronchitis (UID:D001991).

Figure 1: The word cloud represents COVID-19 associated diseases derived through text mining. There are 50 unique major diseases, symptoms and disorders with frequency of appearance ranges from 2 to 552 correspond to font size $1 \%$ and $100 \%$ respectively. The frequencies are highly correlated with the prevalence of comorbid diseases contribute to the severity in COVID-19 patients $\underline{29}$. This consolidated disease network can be readily used by the medical professionals for their regular references.

Figure 2: In the construction of Disease-Disease network, 44 non-isolated diseases (nodes) linked on the basis of disease ontology (DO) based semantic similarity score. The diseases were connected with each other by 89 potential links. The nodes indicate the diseases and node colors on the basis in disease frequency of appearance in the abstracts. The nodes were represented in two shapes circle (diseases) and square (disorders) as well as in three colors yellow (size > 75), green (sizes 75 to 25) and blue (size < 25). The width of the link between diseases represents the similarity score, the significantly comorbid links with (similarity score $>0.5$ ) shown in gray links. The diseases are grouped based on the human system organs and type of disease/disorder (in different background colors with label).

Figure 3: (A) The DO score based clustering of the 44 comorbid diseases of covid-19 were shown in dendrogram. The dendrogram showing five disease clusters namely clusters 1, 2, 3, 4 and 5. (B) In the Heatmap, scaled from 0 to 14, the rows are representing 35 diseases and the column are representing nine topological properties namely Neighborhood, Degree, Eccentricity, Average shortness, topological coefficient, Clustering coefficient, Closeness coefficient, radiality, betweenness of the each disease node. Please note that all the Cluster 1 (pulmonary related diseases) and their symptoms are ranked high in the network in terms of DO association score as well as the network properties (blue backgrounds). (C) Highly comorbid pulmonary diseases of COVID-19 represented in the subnetwork. The colors, shapes of the nodes, edges are as in Figure (2). The highly significant (DO score $>0.5$ ) edges are represented red lines.

\section{Assessment of COVID-19 comorbid respiratory diseases by means of associated genes}


Using CTD, we associated 203 unique genes with 8 comorbid disorders of Cluster 1 . As defined earlier (methods), among 203 genes, 34 genes were involved in interacting with atleast two of the 6 diseases leading to the 79 disease-gene associations. These genes were mainly associated with highly prevalent six diseases of COVID-19 (Fig. 1) excluding the Bronchitis, Pneumothorax and Neonatal asphyxia (Fig. 4). The disease gene relationships were further investigated using disease gene association (DGA) scores derived from various association indices such as Jaccard Index, Simpson Index, Geometric Index and Cosine Index (Supplementry Table 5). The DGA scores revealed that plumonary fibrosis was the most dominant disease in the Cluster 1. In addition, the disease was strongly associated with asthma, Chronic Obstructive Pulmonary Disease (COPD), plumonary edema and ARDS. In the Figure 4, genes strongly

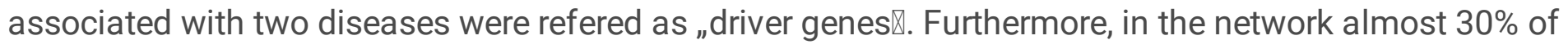
genes (10 genes) were interacting with 3 diseases and revealed the dense disease-gene associations. The functional pathway enrichment of the driver genes revealed the dominant pathway as "oxygen signalling". In addition, our study identified the clinically relevant disease assocation especially ARDS (highiest prevalent disease of COVID-19) strongly associated with plumonary fibrosis through ACE2, CCL2, EDN1, TIMP1 and viral pnuemonia through ACE. These findings highlighted that these 34 genes named "driver genes"could play the major role in the manifestation as well as regulation of the six major diseases/disorders of COVID-19.

Figure 4: The strongly associated pulmonary diseases (D) in red ellipse (second row) and driver genes (G) in green squares of row 3 (G-2D, genes having interaction more than 2 diseases), row 4 (G-3D, genes having interaction more than 3 diseases), row 5 (G-4D, genes having interaction more than 4 diseases), from Cluster 1 is shown as bipartite network. The interaction of each disease with its corresponding genes represented in different color (grey, yellow, violet, pink, blue and black). The number of disease-gene interactions are also mentioned say D-22G, represent the disease asthma interacting with 22 genes and so on. Similarly, the disease- gene-disease association represented in terms of Jaccard score (first row) and the values in square box. Please note the ARDS interacts with viral pneumonia and fibrosis through ACE and ACE2 respectively with the Jaccard score 0.2 .

\section{Tripartite network based prepurposing drugs to treat comorbid diseases of COVID-19}

A drug to be effective against COVID-19 patients in their severe statge should be within or in the immediate interaction with the "COVID-19-target network". We used CTD to extract 505 drugs or chemicals associated with the 6 target network diseases. This expanded network contained a total of 662 disease-drug and 2284 drug-gene connections. We applied the filtering strategy discribed in the Materials and methods section to extract an integrated scale free disease-chemical-gene network. We observed that only few drug nodes with large number of links (Hubs) and the degree distribution followed a power law. The overall network consisted 34 genes, 6 plumonary diseases and 15 drugs (Supplimentry Figure 1). We 
first focused on Food and Drug Administration (FDA) approved drugs namely Dexamethasone, Tretinoin, Acetylcysteine, Oxygen, Simvastatin and Aspirin except the chemical Resveratrol (Fig. 5). Nevertheless, resveratrol is already available as a nutritional supplements in many countries beside its challenges in translation to the clinical drug $\frac{36}{}$. We further investigated whether a network motif analysis could help to prioritize drug targets based on the associations between diseases and their surrounding genes. Based on the " guilt by association" rule - diseases similar to each other are more likely to be affected by the same genes/ pathways, chemicals involved in the same genes are more likely to be highly assoicated with these diseases. For instance, in the network "Plumonary Edema" and "Plumonary fibrosis" shared 15 out of 34 genes. Interestingly, these 15 genes also assoicated with two chemicals "Resveratrol" and "Oxygen" (Fig. 6). Evidently, KEGG pathway enrichment analysis of the 15 genes revealed the key regulation of virus and oncogenic/hypoxic pathways (Fig. 6). This result supported our assoication rule that similar diseases can be treated by same drugs, allowing us to make hypotheses for drugs repositioning purpose. In this analysis, the most densly connected drug hub was "resveratrol" having its association with 4 diseases (out of 6 diseases) and 28 genes (out of 34 genes) (Fig. 5). In this background "Resveratrol" was repositioned for the treatment highly comorbid respiratory disorders of COVID-19 namely asthma, phenmonia, plumonary fibrosis and ARDS.

Figure 5: A tripartite network of pulmonary diseases node (red circles, level 1), drugs node (brown circles, level 2), genes node (green circles, level 3) is shown. The interaction between the different nodes are shown in three color lines. The edges of diseases with drugs and genes are yellow square and green squares respectively. The edges of drugs with diseases and genes are red and blue squares respectively. The numbers inside the squares above and below the circles represent the number of edges of the particular node. Please note that our predicated FDA approved drugs (6) and nutraceutical (1) are strongly interacting with driver genes as well as more than three pulmonary comorbid diseases of COVID-19. Notice the highest number of edges for the drug resveratrol in terms of diseases and genes.

Figure 6: The resveratrol and oxygen chemicals (yellow circle) subgraph of tripartite network is shown (Figure 6). The 15 genes (out of 34 driver genes) highly perturbed by resveratrol drug are shown in green squares. Please note that the same 15 genes also shared by the chemical oxygen (yellow circle). These genes also perturbed during the disease manifestation of pulmonary fibrosis (red ellipse) and pulmonary edema (red ellipse). Notice that the functional enrichment of these genes strongly associate them with major oxygen signaling pathways (HIF, ERK, MAPK, TLR, .. in cyan circle) and leads to the exacerbation of several virus (pink circles) and cancers (violet circles).

\section{Resveratrol significantly reduced hypoxia induced vascular leakage}

The effect of resveratrol on transvascular fluid leakage assessed by quantitation of sodium fluorescein dye leakage (Fig. 7B). After exposure to hypoxia, the mean fluorescein dye leakage $(223.33 \pm 23.60 \mathrm{rfu} / \mathrm{g})$ of lung tissue was significantly higher $(p<0.05)$ as compared to normoxic condition. The animals pre- 
treated with resveratrol $(15 \mathrm{mg} / \mathrm{Kg} \mathrm{BW})$ were exposed to hypoxia showed a significantly lower $(\mathrm{p}<0.05)$ mean relative fluorescence values $(130.75 \pm 5.63 \mathrm{rfu} / \mathrm{g})$ in lungs as compared with hypoxic control (223.34 \pm 23.60$)$ animals. Overall, the administration of resveratrol $(15 \mathrm{mg} / \mathrm{Kg} \mathrm{BW})$ showed a significant decrease in fluorescein dye leakage (lung vascular leakage) in hypoxia exposed animals. However, the rfu values of resveratrol treated hypoxia exposed animals were significantly higher than the normoxic control values.

Figure 7: (A) Rats were injected with drug in their tail vein. (B) Rats were exposed to simulated altitude of 25000 feet $(7620 \mathrm{~m})$ at $22^{\circ} \mathrm{C}$ for $8 \mathrm{~h}$ to observe the effect of Resveratrol $(15 \mathrm{mg} / \mathrm{kg} \mathrm{BW})$ on hypobaric hypoxia- induced vascular permeability. Values are mean \pm SD $(n=6)$. Significant test between groups were determined by using one-way ANOVA followed by Tukey test. * versus Control; \# versus Hypoxia. The experimental conditions were labelled as normoxia $(\mathrm{N})$, normoxia plus drug $(\mathrm{N}+\mathrm{D})$, hypoxia $(\mathrm{H})$ and hypoxia plus drug $(H+D)$

\section{Discussion}

\section{Resveratrol a potential drug to COVID-19 associated comorbid diseases}

The number of hospitalization of COVID-19 patients with one or more comorbid diseases is highly alarming $\underline{29}$. Despite the lack of large clinical data and incomplete understanding of virus pathology, identification of the COVID-19 associated diseases with clinical precision are highly limited. In this regard, our literature-based approach successfully identified broad spectrum of COVID-19 comorbid diseases (54 diseases) and their prevalence on the basis of node size. The DSN is able to accurately capture all the reported broad spectrum of COVID- 19 comorbidities and presented it as a snap shot (Fig. 1). This consolidated disease network can be readily used by the medical professionals for their regular references. The DSN revealed the following important features. (i) Even though severity of the COVID-19 mainly relate to lung dysfunction, the major comorbid diseases are spreading across many vital organs especially heart and kidney. Several clinical reports are coming up in support of our findings also suggesting the same $\underline{23}, \underline{29}, \underline{37}, \underline{38}, \underline{39}, \underline{40}, \underline{41}, \underline{42}, \underline{43}$. (ii) The diseases densely packed yielding high similarity are likely to be comorbid. In this sense, the sub network clusters of respiratory (Cluster1), nephrology (Cluster 2) and cardio respiratory (Cluster 2 \&3) diseases are expected to be comorbid (Fig 3A). As per major clinical studies almost all the disease pairs are proven to be comorbid in COVID-19 patients $\underline{29}, \underline{40}, \underline{4}$. Evidently, every disease node in the respiratory comorbid network share almost $30 \%$ of the entire genes (Fig. 3). Importantly, there are several genes in the host cells utilized by several viruses for their entry as well as replication (Fig. 6). Our study opens up the possibilities that these shared genes also utilized by COVID-19 to establish its virulence against the host cell. In this support, 6 genes associated with COVID19 from literature are common with these driver genes further explains the common pathobiology (Supplementary Figure 2) For instance, COVID-19 could hijack the host cell oxygen signalling pathway genes (15 shared genes) to activate the oxygen deprivation mechanism in lungs (acute lung hypoxia) 
possibly leads to multiple disorders related comorbid symptoms of lung fibrosis, asthma, COPD and ARDS. Furthermore, the genes ACE2 (SARS-COV2 binding target) and ACE may switch the severity from severe (viral pneumonia, lung fibrosis) to critical (ARDS) in COVID-19 patient (Fig. 5). (iii) Instead of treating these comorbid diseases with drugs independently, DSN helped us to prioritize the drugs to treat multiple disorders of COVID-19 (comorbid diseases) simultaneously. Most of our enlisted drugs dexamethasone, acetyl cysteine, and aspirin were showed promising results in their early clinical trials against COVID-19 $45,46,47$. In the rest of drugs resveratrol, Tretinoin and Simvastatin, our study also strongly recommends all of them for the effective treatment of comorbid diseases of COVID-19. For instance, resveratrol is already under clinical trials against the highly comorbid diseases of COVID-19 such as hypertension, diabetics, pneumonia except ARDS and pulmonary edema $\underline{48}, \underline{49}, \underline{50}, \underline{51}$.

\section{Resveratrol may use to reduce vascular leakage in lungs during severe hypoxemia (acute hypoxia) in COVID-19 patients}

Our study clearly resulted out two highly significant findings (i) the single major symptom dominating the entire disease network is lung inflammation. (ii) Resveratrol caused significant reduction of transvascular fluid leakage in lung alveoli as a result of hypoxia insult. The decrease in lung water content by resveratrol are in agreement with animals treated with Tretinoin $\frac{52}{2}, \operatorname{simvastatin} \frac{53}{}$, dexamethasone $\frac{54}{4}$ and acetyl cysteine ${ }^{55}$. Furthermore, the significant reduction in transvascular leakage in the resveratrol treated animals also confirmed using sodium fluorescein dye as an indicator. Similar study design of rats exposed to hypoxia developed acute pulmonary oedema with highly significant increase in the lung fluids, protein like albumin and other vascular compartments $\underline{56}$. The acute pulmonary oedema in humans can be encountered in great variety of conditions $\frac{57}{}$. Importantly, pulmonary oedema could be predisposing to several of the highly comorbid diseases of COVID-19. From our animal experiments it is completely evident that resveratrol $(100 \mathrm{mg} / \mathrm{kg} \mathrm{bw}$ ) considerably reduce the excess accumulation of leverage fluid as the result of acute hypoxic state. In terms of infections, several viral and bacterial infections induced pulmonary inflammation leads excess accumulation of cyokine/chemokines and mucus in the lung alveoli. Several studies it is evident that resveratrol significantly reduce the lung inflammation in infection and thereby suppress the chemokine/cytokine expression in lung alveoli $\underline{58}$. As mentioned earlier, the autopsies of COVID-19 patient \s lung alveoli became stuffed with fluid, white blood cells, mucus and the detritus of destroyed lung cells and completely disrupted the exchange of gases $\underline{26}$. The protective effects of resveratrol in respiratory system may help the COVID-19 patients to breathe easier by decreasing the accumulation of pro-inflammatory molecules, water, chemokines, and cytokines in the lung alveoli. The present study suggested that resveratrol is a potentially useful drug for the treatment of COVID-19 patients by considerably reducing the lung inflammation induced mortality. 


\section{Resveratrol a potential drug to human coronaviruses (CoVs) with caveat to increase ACE2 level}

Most antiviral drugs target specific to viral proteins compromised their efficacy by the rapid evaluation of resistant variants. Consequently, silent information regulator enzymes (SIRT1) of the host cells provide effective therapeutic treatment opportunity to the resistance variants of broad spectrum of viral pathogens ${ }^{\underline{59}}$. Importantly, sirtuin-activating drug resveratrol inhibit the replication of diverse spectrum of viruses $\underline{60-62}$. In addition, resveratrol significantly inhibited MERS-CoV infection and prolonged cellular survival after virus infection $\underline{63}$. In this regard, four 2019-nCoV enzymes that could represent antiviral targets are highly conserved, and share a high level of sequence similarity with the corresponding SARS and MERS $\underline{64,65}$. It is highly reasonable to consider repurposing resveratrol as a selected therapeutic drug for $2019-n C o V \underline{66}$. Instead, the use of resveratrol against severe acute respiratory syndrome coronavirus (SARS-CoV) and SARS-CoV-2 (COVID-19) load resistance needs a careful evaluation by the clinicians. It is evident that resveratrol up regulates the ACE2 in host cells involving Sirt1 enzymes and may facilitate infection with SARS-CoV and SARS-CoV-2 (COVID-19) $\underline{67,68}$. To the surprise, the SARS-CoV-2 (COVID-19) identified with furin cleavage site at the S1/S2 boundary setting the virus distinct from SARS-CoV and SARS- CoVs $\underline{69}$. In the contrary, MERS-CoV is also activated by furin, a serine endoprotease that has been implicated in the processing of fusion proteins and cell entry of other RNA viruses, including HIV, avian influenza A/H5N1 virus, Ebola virus, Zika virus, Marburg virus and flaviviruses $\underline{70}$. Resveratrol potently inhibit almost all the above mentioned viral replications in the host cells but not their entry $\underline{63}, \underline{71}, \underline{72}, \underline{73}, \underline{74}, \underline{75}, \underline{76}, \underline{77}, \underline{78}$. In this regard, we strongly recommend further animal studies are necessary before considering resveratrol for clinical trials.

\section{Nasal administration of resveratrol to control COVID-19 replication in the early stage of infection and to avoid drug metabolism and availability}

COVID-19 infected patients expel virus-laden droplets under the right conditions, liquid droplets from sneezes, coughs and just exhaling. The virus-laden droplets deposit to the lining of the nose and throat of the nearby people who inhale them $\underline{79}, \underline{80}$. COVID-19 is turned out to be the most lethal disease in the world with about $68 \%$ of the mortality are linked to comorbidity 18 . Our study clearly evident that the potential role of resveratrol to control the COVID-19 epidemic. Unfortunately, the therapeutic effect of resveratrol is highly limited due to its poor water solubility, instability, short plasma half-life, and extensive metabolism in the intestine and liver $22,83,84,85$. Therefore, non-invasive parenteral routes must be considered to bring resveratrol to the lungs. Especially for the intranasal deposition of COVID-19 especially in nose and throat during the early stage of infection could be exploited effectively to administrate a sufficient amount of resveratrol. In support of this intranasal administration of resveratrol three times a week for 25 weeks 
resulting in $45 \%$ decrease in tumour volume/mouse ${ }^{86}$. In this study, $200 \mathrm{mM}$ hydroxypropyl- $\beta$-cyclodextrin (HPCD) saline solution is used to solubilize $24 \mathrm{mg} / \mathrm{ml}(105 \mathrm{mM})$ of resveratrol, allowing the administration of a high amount of RES $(1.2 \mathrm{mg} / \mathrm{mouse})$ in a limited volume $(50 \mu \mathrm{l})$ through intranasal instillation $\frac{86}{}$. Another advantage for resveratrol is used as a food supplement in many countries establish the low toxicity $\underline{87}$. Furthermore, the clinical trial of resveratrol carboxymethyl- $\beta$-glucan solution thrice daily during two months in children with allergic rhinitis is capable of significantly improving nasal symptoms in children without any adverse events $\underline{88}$.

\section{Materials And Methods}

\section{Data mining and extraction of bio-entity}

In this study, our goal was to find out the unique relationship between major diseases associated with COVID-19. Pubtator an online text mining tool was used to rank each abstract based on the keyword appearance and annotate the diseases $\underline{89}$. Our version of the PubTator annotations was downloaded on April 23, 2020. Approximately 16.5 million Medline abstracts were searched for the disease keyword "COVID-19", using the Pubtator and the virus keyword severe acute respiratory syndrome coronavirus 2 (SARS-CoV-2) was omitted. The total number of Pubmed Identification numbers (PMIDs) retrieved from the COVID-19 was 6238 as on $23^{\text {rd }}$ April 2020. These PMIDs were submitted to the pubtator to extract high-quality named entity annotations of (1) drugs and other chemicals, (2) genes and (3) diseases related to human from each abstract. The R Package "PubMedWordcloud" was used to construct word cloud based on the disease term frequencies $\underline{90}$. Disease Ontology (DO) score was used to rank the associations among the COVID-19 associated diseases. The R package "DOSE" was used to calculate the DO scores among the diseases obtained from COVID-19 text mining $\underline{91}$. Diseases that missed their links with DO score cut-off $>0.5$ were left out from their disease correlation matrix. The network was constructed in cytoscape 3.6르. The term frequency of each diseases and DO scores between them were added to the COVID-19 comorbid network. The analysis of the generated network was carried out by Cytoscape network analyser to obtain the topological values. The topological values were clustered to narrow down the significant cluster of diseases/disorders using "pvclust" and "pheatmap"package in $\mathrm{R}$ 으, $\underline{94}$.

\section{COVID-19 Comorbid pulmonary Disease-Gene Bipartite Network}

The extraction of genes relationship from Comparative Toxicogenomics Database (CTD) was introduced in the COVID-19 associated pulmonary diseases network as an additional layer of complexity $\underline{95}$. Then, the strength of the relationship of the disease-gene pairs were calculated and represented as a disease-gene association score. The standard association indices include the Jaccard Index, Simpson Index, Geometric index and Cosine index were used for measuring profile similarity in our network with Guide for 
Association Index for Networks webtool (GAIN) $\frac{96}{}$. The similarity between interacting diseases is determined based on the number of shared gene nodes and the total number of diseases connected to these genes. For example, if we define $D g_{1}$ as the set of diseases associated with gene $g_{1}$ and $D g_{2}$ as the set of diseases associated with gene $g_{2} \cdot\left|D g_{1}\right|$ and $\left|D g_{2}\right|$ are the number of diseases associated with $g_{1}$ and $g_{2}$, respectively. $\left|D g_{1} \cap D g_{2}\right|$ indicates the number of shared partners of $D g_{1}$ and $D g_{2}$. The calculations of these indices are summarized in Table (Supplementary Table 5).

\section{Construction of tripartite Network}

To identify potential drug candidates relevant to the bipartite network, we constructed a tripartite network having three communities ( $k=3$ ) i.e. diseases, genes (excluding miRNA) and chemicals. In this tripartite network, all the three communities were closely interconnected to each other such as gene dysfunction may cause one or more core associated diseases that further can be cured by some drugs correcting these gene functions. CTD database was used to get the information regarding associated curated drugs/chemicals with core disease network $\underline{95}$. The Disease-Gene core bipartite network was enriched with the chemicals to construct the tripartite disease-gene-drug/chemical network. The biological networks of

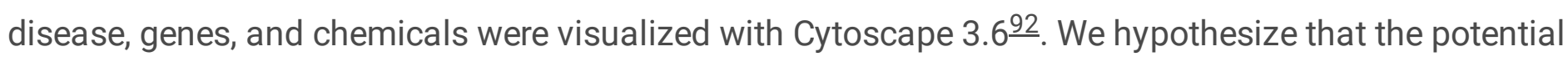
network motifs in the tripartite network can represent basic interrelationships among diseases, drugs, and genes. We filtered out the drugs with minimum $50 \%$ vertices associated to genes and interaction with 3 diseases as well. Specifically, we focus on the three-vertex nodes because they reflect the framework involving all the three communities to achieve particular functions. Out of all possible combinations, we build the tripartite network with potentially relevant interactions comprised of 458 vertices of three different types: 6 diseases, 34 genes and 15 chemicals. Moreover, the tripartite included three different types of interaction edges: about 79 disease-to-gene, over 51 disease-to-chemicals and over 328 genes-tochemicals links (Supplementary Figure 1). Kyoto Encyclopedia of Genes and Genomes (KEGG) pathway based gene set enrichment analysis was carried out for the 34 genes of interest $\underline{97}$.

\section{Ethical approval}

Animal Ethical Committee of the Institute in accordance with Committee for the Purpose of Control and Supervision of Experiments on Animals (CPCSEA), of the Government of India has approved the study (IAEC No. DIPAS/ IAEC/ 2017/ 18). The ARRIVE (Animal Research: Reporting of In Vivo Experiments) guidelines for reporting animal research was followed $\underline{98}$. 


\section{Animal Experiments}

In all our study, male Sprague-Dawley (SD) rats (200-220 g) were used. The animals were maintained in the Institute animal house (Animal facility, DIPAS) at $23^{\circ} \mathrm{C} \pm 2{ }^{\circ} \mathrm{C}$ with a $12-12 \mathrm{~h}$ day-night cycle with suitable accessibility of nourishment. The experiments were carried out following the approval and guidelines of the ethics committee of the institute. The rats were randomly subdivided into 4 batches containing four experimental groups with replicates (n) of 6 in each group (Supplementary Figure 3). Animals from hypoxic groups were exposed to a simulated altitude of 25000 feet $(7620 \mathrm{~m})$ in animal decompression chamber at $22^{\circ} \mathrm{C}$ (Decibel Instruments, India) coupled to a mercury barometer for $8 \mathrm{~h} \underline{99}$. The airflow in the chamber was $2 \mathrm{~L} / \mathrm{min}$ with relative humidity maintained at $50-55 \%$. After exposure to hypoxia, the animals were anesthetized using ketamine $(80 \mathrm{mg} / \mathrm{kg})$ and xylazine $(20 \mathrm{mg} / \mathrm{kg})$, lungs were dissected out enbloc after perfusion with ice-cold phosphate buffer saline (PBS) to remove the blood and snap frozen at $-80^{\circ} \mathrm{C}$ for further analysis.

\section{Determination of vascular permeability}

The permeability assays were performed by the method of Purushothaman et al, using sodium fluorescein dye (Sigma, USA) extravasation as an indicator of vascular leakage $\frac{56}{6}$ after exposing the rats for $8 \mathrm{~h}$ of hypoxia. Rat tail vein were injected with sodium fluorescein dye (15mg/kg/BW) in PBS (Fig. 7A). The injected rats were anesthetized after half an hour of dye injection. The rats were perfused through the left ventricle with $30 \mathrm{~mL}$ of phosphate- buffered saline to remove the fluorescein from the vascular bed. The lung was harvested from the rats for further studies. The fluorescein dye permeability in lung was measured at an excitation wavelength of $485 \mathrm{~nm}$ and an emission wavelength of $531 \mathrm{~nm}$ using a Spectro fluorimeter (FLUO star Omega). The lung weight was measured and incubated in formamide for 16 hours to extract sodium fluorescein $\frac{100}{}$. The absorption values were obtained as relative fluorescence units (rfu) per gram dry weight of the tissue. The relative percentage change in fluorescein absorbance for the treatment groups 2, 3 and 4 were calculated in comparison with control group 1 (normoxic state).

\section{References}

1. Coronavirus disease 2019 (COVID-19). Situation Report - 94 (2020).

2. , I. M. F. R. World Economic Outlook, April 2020 : The Great Lockdown. (April 14, 2020).

3. Modes of transmission of virus causing COVID-19:implications for IPC precaution recommendations. Scientific Brief (2020).

4. Coronavirus disease (COVID-19) advice for the public.

5. Lewis, D. Is the coronavirus airborne? Experts can't agree. Nature 580, 175, doi:10.1038/d41586-02000974-w (2020). 
6. Zou, L. et al. SARS-CoV-2 Viral Load in Upper Respiratory Specimens of Infected Patients. The New England journal of medicine 382, 1177-1179, doi:10.1056/NEJMc2001737 (2020).

7. Lipsitch, M., Swerdlow, D. L. \& Finelli, L. Defining the Epidemiology of Covid-19 - Studies Needed. The New England journal of medicine 382, 1194-1196, doi:10.1056/NEJMp2002125 (2020).

8. He, X. et al. Temporal dynamics in viral shedding and transmissibility of COVID-19. Nature medicine, doi:10.1038/s41591-020-0869-5 (2020).

9. Siordia, J. A., Jr. Epidemiology and clinical features of COVID-19: A review of current literature. Journal of clinical virology : the official publication of the Pan American Society for Clinical Virology 127, 104357, doi:10.1016/j.jcv.2020.104357 (2020).

10. Hijano, D. R., Maron, G. \& Hayden, R. T. Respiratory Viral Infections in Patients With Cancer or Undergoing Hematopoietic Cell Transplant. Frontiers in microbiology 9, 3097, doi:10.3389/fmicb.2018.03097 (2018).

11. Galvan, J. M., Rajas, O. \& Aspa, J. Review of Non-Bacterial Infections in Respiratory Medicine: Viral Pneumonia. Archivos de bronconeumologia 51, 590-597, doi:10.1016/j.arbres.2015.02.015 (2015).

12. Fehr, R. \& Perlman, S. Coronaviruses: an overview of their replication and pathogenesis. Methods in molecular biology 1282, 1-23, doi:10.1007/978-1-4939-2438-7_1 (2015).

13. Khomich, O. A., Kochetkov, S. N., Bartosch, B. \& Ivanov, A. V. Redox Biology of Respiratory Viral Infections. Viruses 10, doi:10.3390/v10080392 (2018).

14. Ramalingam, S., Graham, C., Dove, J., Morrice, L. \& Sheikh, A. A pilot, open labelled, randomised controlled trial of hypertonic saline nasal irrigation and gargling for the common cold. Scientific reports 9, 1015, doi:10.1038/s41598-018-37703-3 (2019).

15. Muktibodhananda, S. Hatha Yoga Pradipika. Light on Hatha Yoga. Bihar School of Yoga, India 4th Edition, 202-205 (2012).

16. Liu, Y. et al. Viral dynamics in mild and severe cases of COVID-19. The Lancet. Infectious diseases, doi:10.1016/S1473-3099(20)30232-2 (2020).

17. Wang, M. et al. Remdesivir and chloroquine effectively inhibit the recently emerged novel coronavirus (2019-nCoV) in vitro. Cell research 30, 269-271, doi:10.1038/s41422-020-0282-0 (2020).

18. Cao, B. et al. A Trial of Lopinavir-Ritonavir in Adults Hospitalized with Severe Covid-19. The New England journal of medicine, doi:10.1056/NEJMoa2001282 (2020).

19. Duan, Y. J. et al. The Trial of Chloroquine in the Treatment of Corona Virus Disease 2019 COVID-19 and Its Research Progress in Forensic Toxicology. Fa yi xue za zhi 36, doi:10.12116/j.issn.10045619.2020.02.001 (2020).

20. Molina, J. M. et al. No evidence of rapid antiviral clearance or clinical benefit with the combination of hydroxychloroquine and azithromycin in patients with severe COVID-19 infection. Medecine et maladies infectieuses, doi:10.1016/j.medmal.2020.03.006 (2020).

21. Cologne, I. f. Q. a. E. i. H. C. I. How are different types of pneumonia classified? InformedHealth.org [Internet]. NBK525768 (2006). 
22. Md Insiat Islam, R. Current Drugs with Potential for Treatment of COVID-19: A Literature Review. Journal of pharmacy \& pharmaceutical sciences : a publication of the Canadian Society for Pharmaceutical Sciences, Societe canadienne des sciences pharmaceutiques 23, 58-64, doi:10.18433/jpps31002 (2020).

23. Zhou, F. et al. Clinical course and risk factors for mortality of adult inpatients with COVID-19 in Wuhan, China: a retrospective cohort study. Lancet 395, 1054-1062, doi:10.1016/S01406736(20)30566-3 (2020).

24. Wu, C. et al. Risk Factors Associated With Acute Respiratory Distress Syndrome and Death in Patients With Coronavirus Disease 2019 Pneumonia in Wuhan, China. JAMA internal medicine, doi:10.1001/jamainternmed.2020.0994 (2020).

25. Gattinoni, L. et al. COVID-19 pneumonia: different respiratory treatments for different phenotypes? Intensive care medicine, doi:10.1007/s00134-020-06033-2 (2020).

26. Meredith Wadman, J. C.-F., Jocelyn Kaiser, Catherine Matacic. How does coronavirus kill? Clinicians trace a ferocious rampage through the body, from brain to toes. Science, doi:https://doi.org/10.1126/science.abc3208 (2020).

27. Richardson, S. et al. Presenting Characteristics, Comorbidities, and Outcomes Among 5700 Patients Hospitalized With COVID-19 in the New York City Area. Jama, doi:10.1001/jama.2020.6775 (2020).

28. Yang, J. et al. Prevalence of comorbidities in the novel Wuhan coronavirus (COVID-19) infection: a systematic review and meta-analysis. International journal of infectious diseases : IJID : official publication of the International Society for Infectious Diseases, doi:10.1016/j.ijid.2020.03.017 (2020).

29. Guan, W. J. et al. Comorbidity and its impact on 1590 patients with Covid-19 in China: A Nationwide Analysis. The European respiratory journal, doi:10.1183/13993003.00547-2020 (2020).

30. Meng, et al. Renin-angiotensin system inhibitors improve the clinical outcomes of COVID- 19 patients with hypertension. Emerging microbes \& infections 9, 757-760, doi:10.1080/22221751.2020.1746200 (2020).

31. Wong, N. D. et al. Spotlight from the American Society for Preventive Cardiology on Key Features of the 2018 AHA/ACC/AACVPR/AAPA/ABC/ACPM/ADA/AGS/APhA/ASPC/NLA/PCNA Guidelines on the Management of Blood Cholesterol. American journal of cardiovascular drugs : drugs, devices, and other interventions 20, 1-9, doi:10.1007/s40256-019-00358-0 (2020).

32. Singh, A. K., Gupta, R. \& Misra, A. Comorbidities in COVID-19: Outcomes in hypertensive cohort and controversies with renin angiotensin system blockers. Diabetes \& metabolic syndrome 14, 283-287, doi:10.1016/j.dsx.2020.03.016 (2020).

33. Vuille-dit-Bille, R. N. et al. Human intestine luminal ACE2 and amino acid transporter expression increased by ACE-inhibitors. Amino acids 47, 693-705, doi:10.1007/s00726-014- 1889-6 (2015).

34. Fang, L., Karakiulakis, G. \& Roth, M. Antihypertensive drugs and risk of COVID-19? - Authors' reply. The Lancet. Respiratory medicine, doi:10.1016/S2213-2600(20)30159-4 (2020).

35. Baumann, N. How to use the medical subject headings (MeSH). International journal of clinical practice 70, 171-174, doi:10.1111/ijcp.12767 (2016). 
36. Subramanian, L. et al. Resveratrol: challenges in translation to the clinic--a critical discussion. Clinical cancer research : an official journal of the American Association for Cancer Research 16, 5942-5948, doi:10.1158/1078-0432.CCR-10-1486 (2010).

37. Shi, S. et al. Association of Cardiac Injury With Mortality in Hospitalized Patients With COVID- 19 in Wuhan, China. JAMA cardiology, doi:10.1001/jamacardio.2020.0950 (2020).

38. Wang, D. et al. Clinical Characteristics of 138 Hospitalized Patients With 2019 Novel CoronavirusInfected Pneumonia in Wuhan, China. Jama, doi:10.1001/jama.2020.1585 (2020).

39. Li, B. et al. Prevalence and impact of cardiovascular metabolic diseases on COVID-19 in China. Clinical research in cardiology : official journal of the German Cardiac Society, doi:10.1007/s00392020-01626-9 (2020).

40. Yang, J. et al. Prevalence of comorbidities and its effects in coronavirus disease 2019 patients: A systematic review and meta-analysis. International journal of infectious diseases : IJID : official publication of the International Society for Infectious Diseases 94, 91-95, doi:10.1016/j.ijid.2020.03.017 (2020).

41. Zhang, Y. et al. [Clinical and coagulation characteristics of 7 patients with critical COVID-2019 pneumonia and acro-ischemia]. Zhonghua xue ye xue za zhi = Zhonghua xueyexue zazhi 41, E006, doi:10.3760/cma.j.issn.0253-2727.2020.0006 (2020).

42. Wang, et al. Comorbidities and multi-organ injuries in the treatment of COVID-19. Lancet 395, e52, doi:10.1016/S0140-6736(20)30558-4 (2020).

43. Cheng, Y. et al. Kidney disease is associated with in-hospital death of patients with COVID-19. Kidney international, doi:10.1016/j.kint.2020.03.005 (2020).

44. Emami, A., Javanmardi, F., Pirbonyeh, N. \& Akbari, A. Prevalence of Underlying Diseases in Hospitalized Patients with COVID-19: a Systematic Review and Meta-Analysis. Archives of academic emergency medicine 8, e35 (2020).

45. Al Saleh, A. S., Sher, T. \& Gertz, M. A. Multiple Myeloma in the Time of COVID-19. Acta haematologica, 1-7, doi:10.1159/000507690 (2020).

46. Horowitz, R. I., Freeman, P. R. \& Bruzzese, J. Efficacy of glutathione therapy in relieving dyspnea associated with COVID-19 pneumonia: A report of 2 cases. Respiratory medicine case reports, 101063, doi:10.1016/j.rmcr.2020.101063 (2020).

47. Kwiatkowski, S. et al. Why we should not stop giving aspirin to pregnant women during the COVID-19 pandemic. Ultrasound in obstetrics \& gynecology : the official journal of the International Society of Ultrasound in Obstetrics and Gynecology, doi:10.1002/uog.22049 (2020).

48. Liu, W., Chen, P., Deng, J., Lv, J. \& Liu, J. Resveratrol and polydatin as modulators of Ca(2+) mobilization in the cardiovascular system. Annals of the New York Academy of Sciences 1403, 82-91, doi:10.1111/nyas.13386 (2017).

49. Zordoky, B. N., Robertson, I. M. \& Dyck, J. R. Preclinical and clinical evidence for the role of resveratrol in the treatment of cardiovascular diseases. Biochimica et biophysica acta 1852, 1155-1177, doi:10.1016/j.bbadis.2014.10.016 (2015). 
50. Hoseini, A. et al. The effects of resveratrol on metabolic status in patients with type 2 diabetes mellitus and coronary heart disease. Food \& function 10, 6042-6051, doi:10.1039/c9fo01075k (2019).

51. Qiang, L., Di, Y., Jiang, Z. \& Xu, J. Resveratrol improves efficacy of oral amoxicillin against childhood fast breathing pneumonia in a randomized placebo-controlled double blind clinical trial. Microbial pathogenesis 114, 209-212, doi:10.1016/j.micpath.2017.11.062 (2018).

52. Lucas, R. et al. Agonist of growth hormone-releasing hormone reduces pneumolysin-induced pulmonary permeability edema. Proceedings of the National Academy of Sciences of the United States of America 109, 2084-2089, doi:10.1073/pnas.1121075109 (2012).

53. Bao, X. C. et al. Simvastatin decreases hyperbaric oxygen-induced acute lung injury by upregulating eNOS. American journal of physiology. Lung cellular and molecular physiology 314, L287-L297, doi:10.1152/ajplung.00520.2016 (2018).

54. Sikri, G. Role of dexamethasone in prevention of high altitude pulmonary edema. Journal of occupational health 57, 200, doi:10.1539/joh.L-14-0002 (2015).

55. Campos, R. et al. N-acetylcysteine prevents pulmonary edema and acute kidney injury in rats with sepsis submitted to mechanical ventilation. American journal of physiology. Lung cellular and molecular physiology 302, L640-650, doi:10.1152/ajplung.00097.2011 (2012).

56. Purushothaman, J. et al. Modulation of Hypoxia-Induced Pulmonary Vascular Leakage in Rats by Seabuckthorn (Hippophae rhamnoides L.). Evidence-based complementary and alternative medicine : eCAM 2011, 574524, doi:10.1093/ecam/nep199 (2011).

57. Luisada, A. A. \& Cardi, L. Acute pulmonary edema; pathology, physiology and clinical management. Circulation 13, 113-135, doi:10.1161/01.cir.13.1.113 (1956).

58. Knobloch, J. et al. Resveratrol attenuates the release of inflammatory cytokines from human bronchial smooth muscle cells exposed to lipoteichoic acid in chronic obstructive pulmonary disease. Basic \& clinical pharmacology \& toxicology 114, 202-209, doi:10.1111/bcpt.12129 (2014).

59. Koyuncu, E. et al. Sirtuins are evolutionarily conserved viral restriction factors. mBio 5, doi:10.1128/mBio.02249-14 (2014).

60. Yang, T. et al. Resveratrol, sirtuins, and viruses. Reviews in medical virology 25, 431-445, doi:10.1002/rmv.1858 (2015).

61. Mohar, D. S. \& Malik, S. The Sirtuin System: The Holy Grail of Resveratrol? Journal of clinical \& experimental cardiology 3, doi:10.4172/2155-9880.1000216 (2012).

62. Li, H., Xia, N., Hasselwander, S. \& Daiber, A. Resveratrol and Vascular Function. International journal of molecular sciences 20, doi:10.3390/ijms20092155 (2019).

63. Lin, S. C. et al. Effective inhibition of MERS-CoV infection by resveratrol. BMC infectious diseases 17, 144, doi:10.1186/s12879-017-2253-8 (2017).

64. Liu, C. et al. Research and Development on Therapeutic Agents and Vaccines for COVID-19 and Related Human Coronavirus Diseases. ACS central science 6, 315-331, doi:10.1021/acscentsci.0c00272 (2020). 
65. Hoffmann, M. et al. SARS-CoV-2 Cell Entry Depends on ACE2 and TMPRSS2 and Is Blocked by a Clinically Proven Protease Inhibitor. Cell 181, 271-280 e278, doi:10.1016/j.cell.2020.02.052 (2020).

66. Li, G. \& De Clercq, E. Therapeutic options for the 2019 novel coronavirus (2019-nCoV). Nature reviews. Drug discovery 19, 149-150, doi:10.1038/d41573-020-00016-0 (2020).

67. Moran, C. S. et al. Resveratrol Inhibits Growth of Experimental Abdominal Aortic Aneurysm Associated With Upregulation of Angiotensin-Converting Enzyme 2. Arteriosclerosis, thrombosis, and vascular biology 37, 2195-2203, doi:10.1161/ATVBAHA.117.310129 (2017).

68. Jang, A. et al. Effects of Resveratrol on the Renin-Angiotensin System in the Aging Kidney. Nutrients 10, doi:10.3390/nu10111741 (2018).

69. Walls, A. C. et al. Structure, Function, and Antigenicity of the SARS-CoV-2 Spike Cell 181, 281-292 e286, doi:10.1016/j.cell.2020.02.058 (2020).

70. Zumla, A., Chan, J. F., Azhar, E. I., Hui, D. S. \& Yuen, K. Y. Coronaviruses - drug discovery and therapeutic options. Nature reviews. Drug discovery 15, 327-347, doi:10.1038/nrd.2015.37 (2016).

71. Chan, C. N., Trinite, B. \& Levy, D. N. Potent Inhibition of HIV-1 Replication in Resting CD4 T Cells by Resveratrol and Pterostilbene. Antimicrobial agents and chemotherapy 61, doi:10.1128/AAC.0040817 (2017).

72. Friel, H. \& Lederman, H. A nutritional supplement formula for influenza A (H5N1) infection in humans. Medical hypotheses 67, 578-587, doi:10.1016/j.mehy.2006.02.040 (2006).

73. Mohd, A., Zainal, N., Tan, K. K. \& AbuBakar, S. Resveratrol affects Zika virus replication in vitro. Scientific reports 9, 14336, doi:10.1038/s41598-019-50674-3 (2019).

74. Evers, D. L., Wang, X., Huong, S. M., Huang, D. Y. \& Huang, E. S. 3,4',5-Trihydroxy-trans- stilbene (resveratrol) inhibits human cytomegalovirus replication and virus-induced cellular signaling. Antiviral research 63, 85-95, doi:10.1016/j.antiviral.2004.03.002 (2004).

75. Berardi, V., Ricci, F., Castelli, M., Galati, G. \& Risuleo, G. Resveratrol exhibits a strong cytotoxic activity in cultured cells and has an antiviral action against polyomavirus: potential clinical use. Journal of experimental \& clinical cancer research : CR 28, 96, doi:10.1186/1756- 9966-28-96 (2009).

76. De Leo, A. et al. Resveratrol inhibits Epstein Barr Virus lytic cycle in Burkitt's lymphoma cells by affecting multiple molecular targets. Antiviral research 96, 196-202, doi:10.1016/j.antiviral.2012.09.003 (2012).

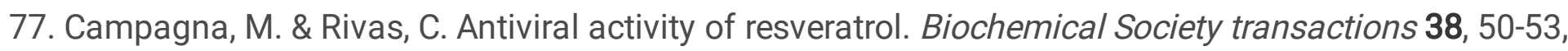
doi:10.1042/BST0380050 (2010).

78. Zhao, X. et al. Antiviral properties of resveratrol against pseudorabies virus are associated with the inhibition of IkappaB kinase activation. Scientific reports 7, 8782, doi:10.1038/s41598-017-09365-0 (2017).

79. Sims, A. C., Burkett, S. E., Yount, B. \& Pickles, R. J. SARS-CoV replication and pathogenesis in an in vitro model of the human conducting airway epithelium. Virus research 133, 33-44, doi:10.1016/j.virusres.2007.03.013 (2008). 
80. Aleksandra Milewskaa, A. K.-P., Jakub Wadasb, Agnieszka Sudera, Artur Szczepanskia, Agnieszka Dabrowska, Katarzyna Owczareka, Marek Ochmanc , Tomasz Stacelc, Zenon Rajfurd, Pawel Labaja, Wojciech Branickia, Krzysztof Pyrca. Replication of SARS-CoV-2 in human respiratory epithelium. doi:10.1101/2020.03.20.999029 (2020).

81. Wang, B., Li, R., Lu, Z. \& Huang, Y. Does comorbidity increase the risk of patients with COVID- 19: evidence from meta-analysis. Aging 12, 6049-6057, doi:10.18632/aging.103000 (2020).

82. Amri, A., Chaumeil, J. C., Sfar, S. \& Charrueau, C. Administration of resveratrol: What formulation solutions to bioavailability limitations? Journal of controlled release : official journal of the Controlled Release Society 158, 182-193, doi:10.1016/j.jconrel.2011.09.083 (2012).

83. Marier, J. F. et al. Metabolism and disposition of resveratrol in rats: extent of absorption, glucuronidation, and enterohepatic recirculation evidenced by a linked-rat model. The Journal of pharmacology and experimental therapeutics 302, 369-373, doi:10.1124/jpet.102.033340 (2002).

84. Berman, A. Y., Motechin, R. A., Wiesenfeld, M. Y. \& Holz, M. K. The therapeutic potential of resveratrol: a review of clinical trials. NPJ precision oncology 1, doi:10.1038/s41698-017-0038-6 (2017).

85. Gambini, J. et al. Properties of Resveratrol: In Vitro and In Vivo Studies about Metabolism, Bioavailability, and Biological Effects in Animal Models and Humans. Oxidative medicine and cellular longevity 2015, 837042, doi:10.1155/2015/837042 (2015).

86. Monteillier, A., Voisin, A., Furrer, P., Allemann, E. \& Cuendet, M. Intranasal administration of resveratrol successfully prevents lung cancer in A/J mice. Scientific reports 8, 14257, doi:10.1038/s41598-01832423-0 (2018).

87. Salehi, B. et al. Resveratrol: A Double-Edged Sword in Health Benefits. Biomedicines 6, doi:10.3390/biomedicines6030091 (2018).

88. Miraglia Del Giudice, M. et al. Resveratrol plus carboxymethyl-beta-glucan reduces nasal symptoms in children with pollen-induced allergic rhinitis. Current medical research and opinion 30, 1931-1935, doi:10.1185/03007995.2014.938731 (2014).

89. Wei, C. H., Allot, A., Leaman, R. \& Lu, Z. PubTator central: automated concept annotation for biomedical full text articles. Nucleic acids research 47, W587-W593, doi:10.1093/nar/gkz389 (2019).

90. Fan, F. Y. PubMedWordcloud: 'Pubmed' Word Clouds. package version 0.3.6 (2019).

91. Yu, G., Wang, L. G., Yan, G. R. \& He, Q. Y. DOSE: an R/Bioconductor package for disease ontology semantic and enrichment analysis. Bioinformatics 31, 608-609, doi:10.1093/bioinformatics/btu684 (2015).

92. Shannon, P. et al. Cytoscape: a software environment for integrated models of biomolecular interaction networks. Genome research 13, 2498-2504, doi:10.1101/gr.1239303 (2003).

93. Shimodaira, R. S. a. Y. T. a. H. \{pvclust: Hierarchical Clustering with P-Values via Multiscale Bootstrap Resampling. R package version 2.2-0 (2019).

94. Kolde, R. pheatmap: Pretty Heatmaps. $R$ package version 1.0.12 (2019).

95. Davis, A. P. et al. The Comparative Toxicogenomics Database: update 2019. Nucleic acids research 47, D948-D954, doi:10.1093/nar/gky868 (2019). 
96. Fuxman Bass, J. I. et al. Using networks to measure similarity between genes: association index selection. Nature methods 10, 1169-1176, doi:10.1038/nmeth.2728 (2013).

97. Kanehisa, M. \& Goto, S. KEGG: kyoto encyclopedia of genes and genomes. Nucleic acids research 28, 27-30, doi:10.1093/nar/28.1.27 (2000).

98. Kilkenny, C., Browne, W. J., Cuthill, I. C., Emerson, M. \& Altman, D. G. Improving bioscience research reporting: the ARRIVE guidelines for reporting animal research. PLoS biology 8, e1000412, doi:10.1371/journal.pbio.1000412 (2010).

99. Sarada, et al. Role of oxidative stress and NFkB in hypoxia-induced pulmonary edema. Experimental biology and medicine 233, 1088-1098, doi:10.3181/0712-RM-337 (2008).

100. Jacob, J. S., Rosen, E. S. \& Young, E. Report on the presence of a toxic substance, dimethyl formamide, in sodium fluorescein used for fluorescein angiography. The British journal of ophthalmology 66, 567-568, doi:10.1136/bjo.66.9.567 (1982).

\section{Declarations}

Acknowledgements

Not applicable

Author information Affiliations

Defence Institute of Physiology and Allied Sciences, Defence Research and Development Organization, Lucknow Road, Timarpur, Delhi, India

Jai Chand Patel, Rajkumar Tulswani, Pankaj Khurana, Yogendra Kumar Sharma, Lilly Ganju, Bhuvnesh Kumar and Ragumani Sugadev ${ }^{*}$

Contributions

RS and JCP conceptualized, data collected/analysed, result visualized and drafted the manuscript for this study. PK, YKS, LG and BK analysis and drafting the manuscript. All authors have read and approved the final manuscript.

Funding

There is no funding source for this research work. 
Corresponding author

Correspondence to Ragumani Sugadev.

Ethics declarations Competing interests

The authors declare that they have no competing interests.

Consent for publication

Not Applicable.

\section{Figures}

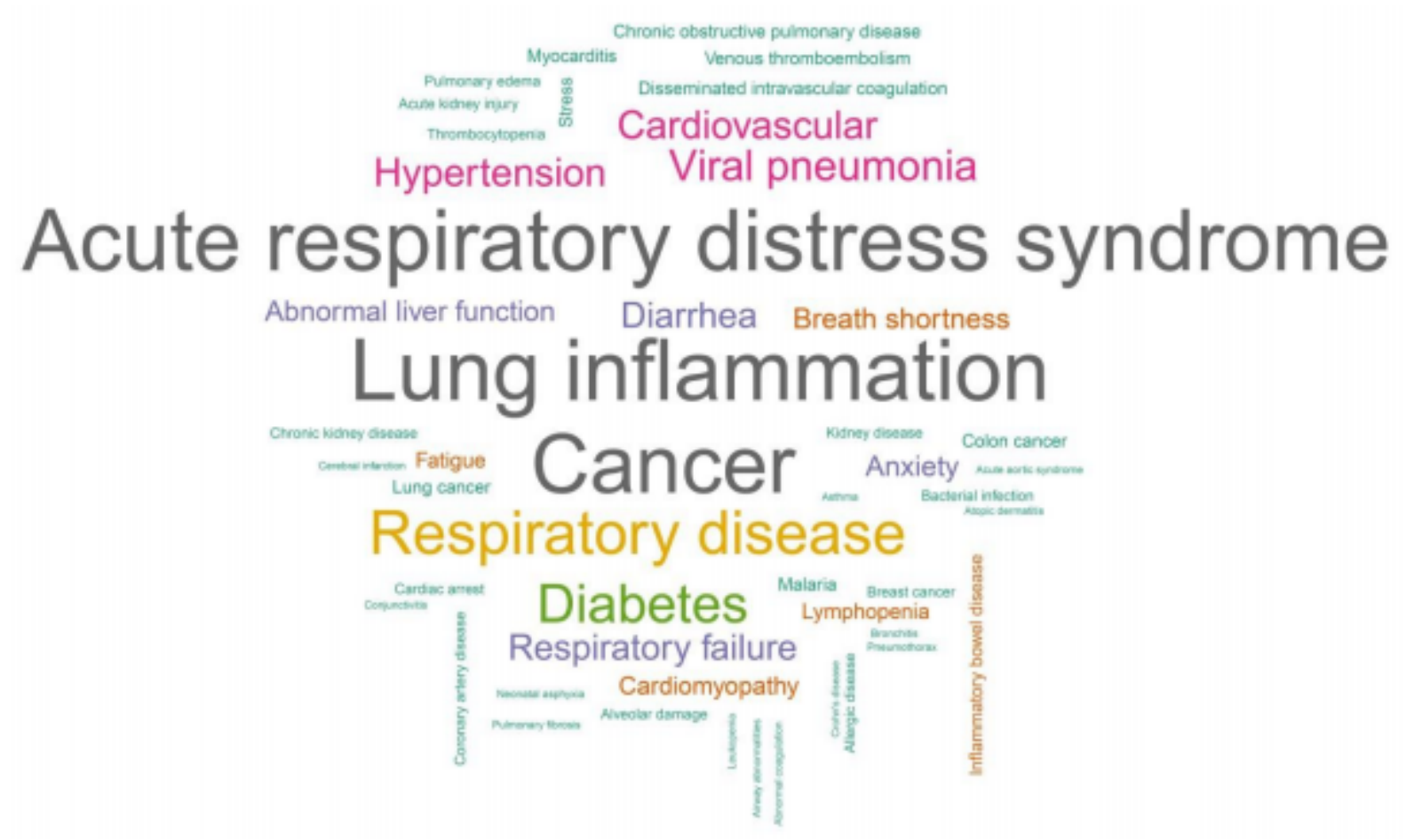

\section{Figure 1}

The word cloud represents COVID-19 associated diseases derived through text mining. There are 50 unique major diseases, symptoms and disorders with frequency of appearance ranges from 2 to 552 correspond to font size $1 \%$ and $100 \%$ respectively. The frequencies are highly correlated with the prevalence of comorbid diseases contribute to the severity in COVID-19 patients29. This consolidated disease network can be readily used by the medical professionals for their regular references. 


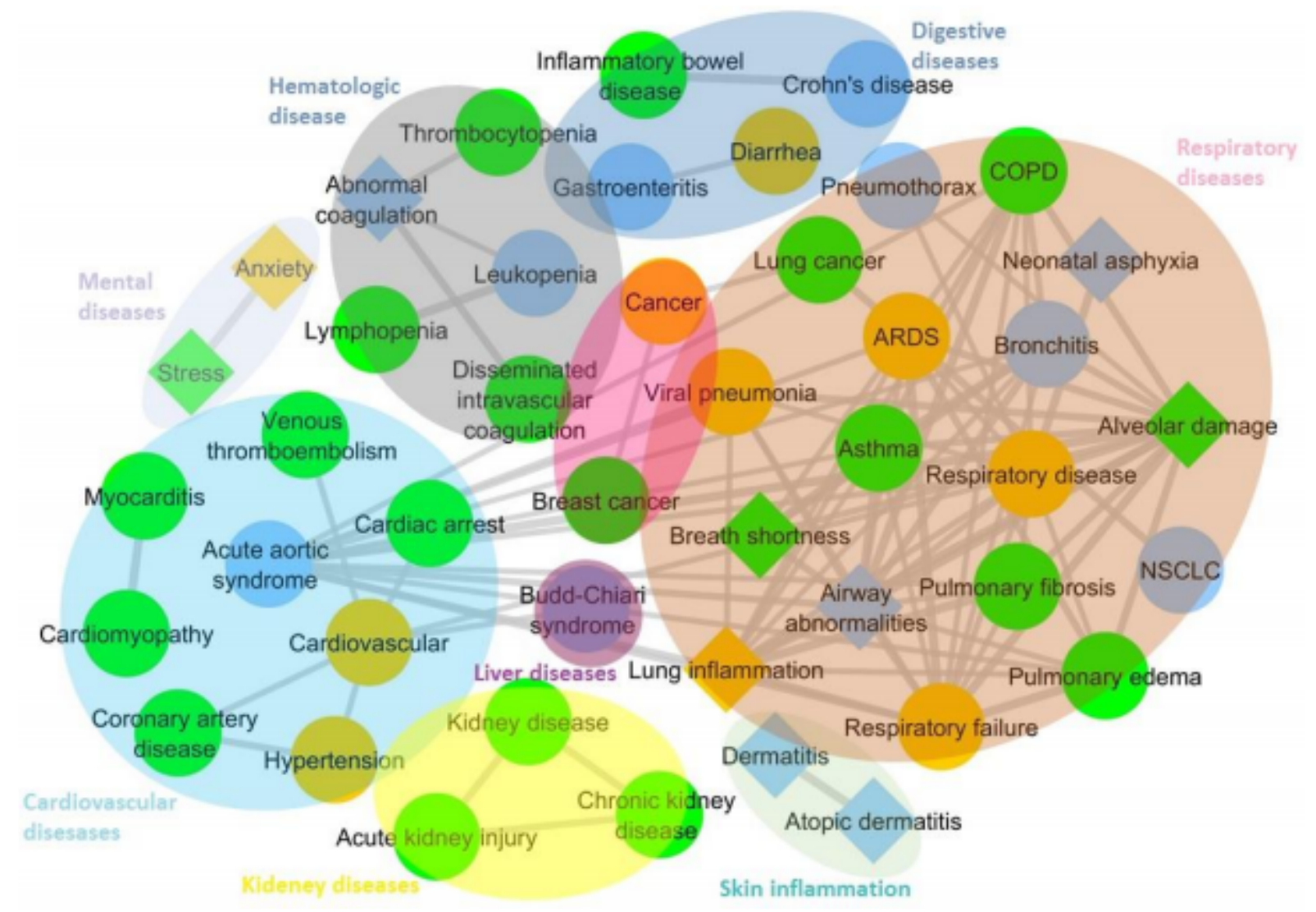

Figure 2

In the construction of Disease-Disease network, 44 non-isolated diseases (nodes) linked on the basis of disease ontology (DO) based semantic similarity score. The diseases were connected with each other by 89 potential links. The nodes indicate the diseases and node colors on the basis in disease frequency of appearance in the abstracts. The nodes were represented in two shapes circle (diseases) and square (disorders) as well as in three colors yellow (size > 75), green (sizes 75 to 25) and blue (size < 25). The width of the link between diseases represents the similarity score, the significantly comorbid links with (similarity score $>0.5$ ) shown in gray links. The diseases are grouped based on the human system organs and type of disease/disorder (in different background colors with label). 
A

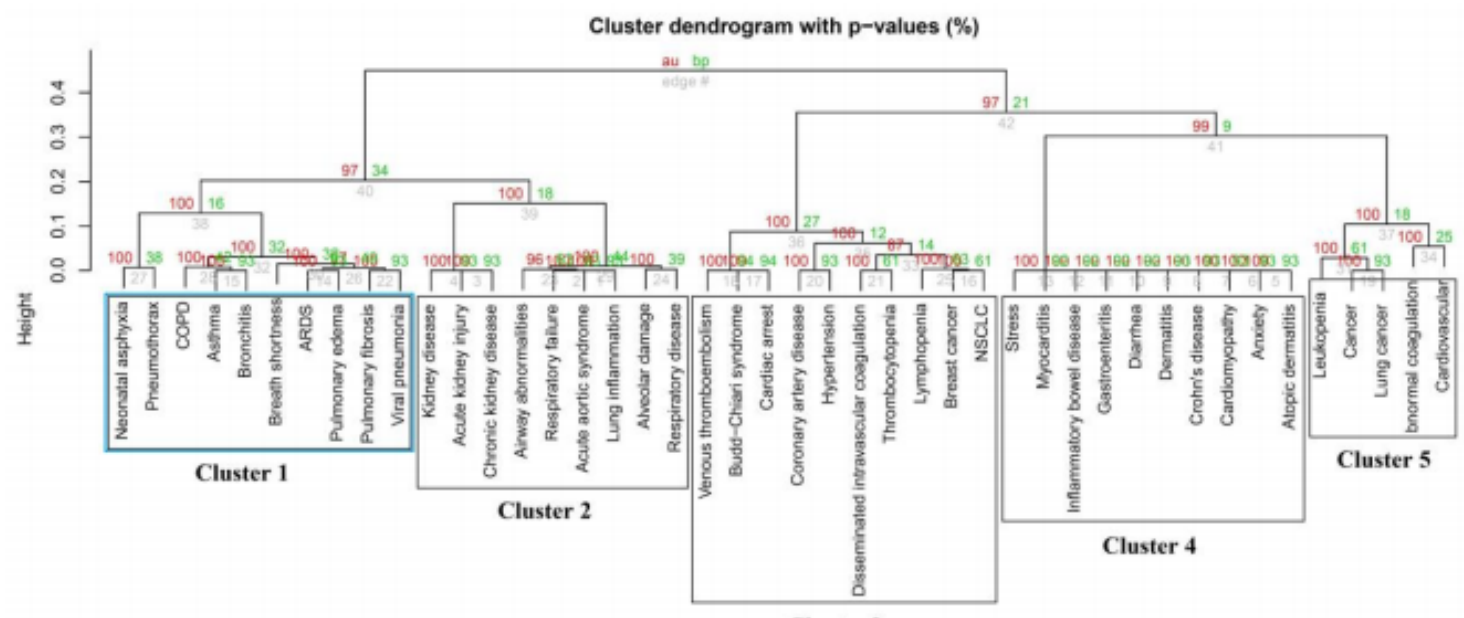

Cluster 3

B

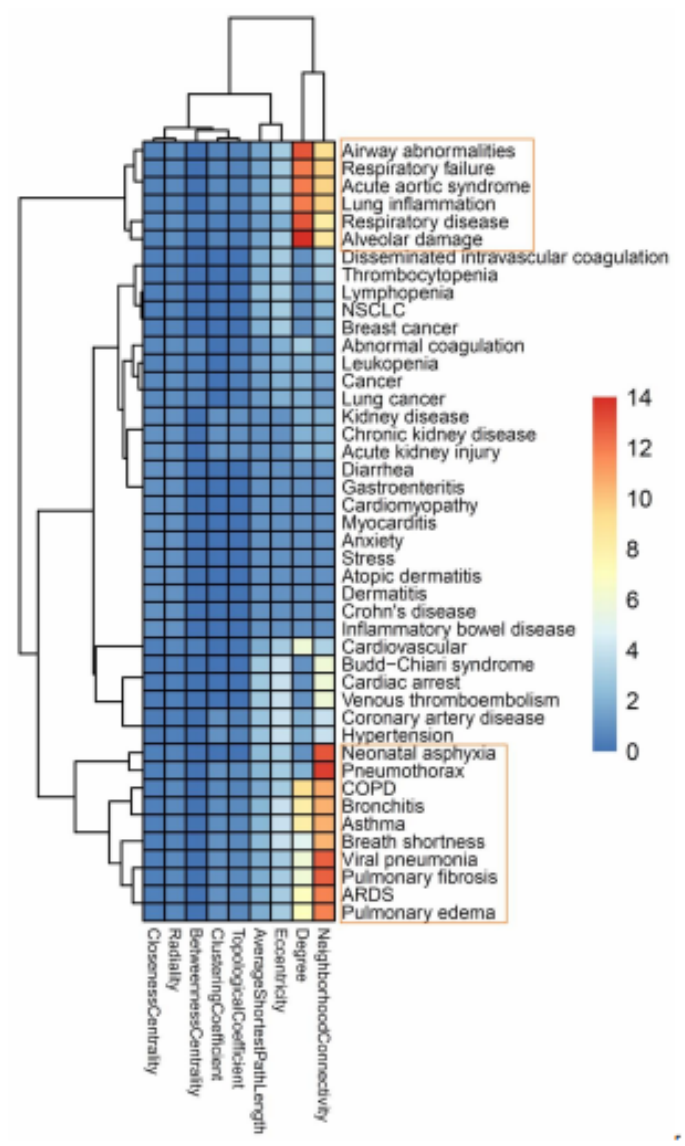

C

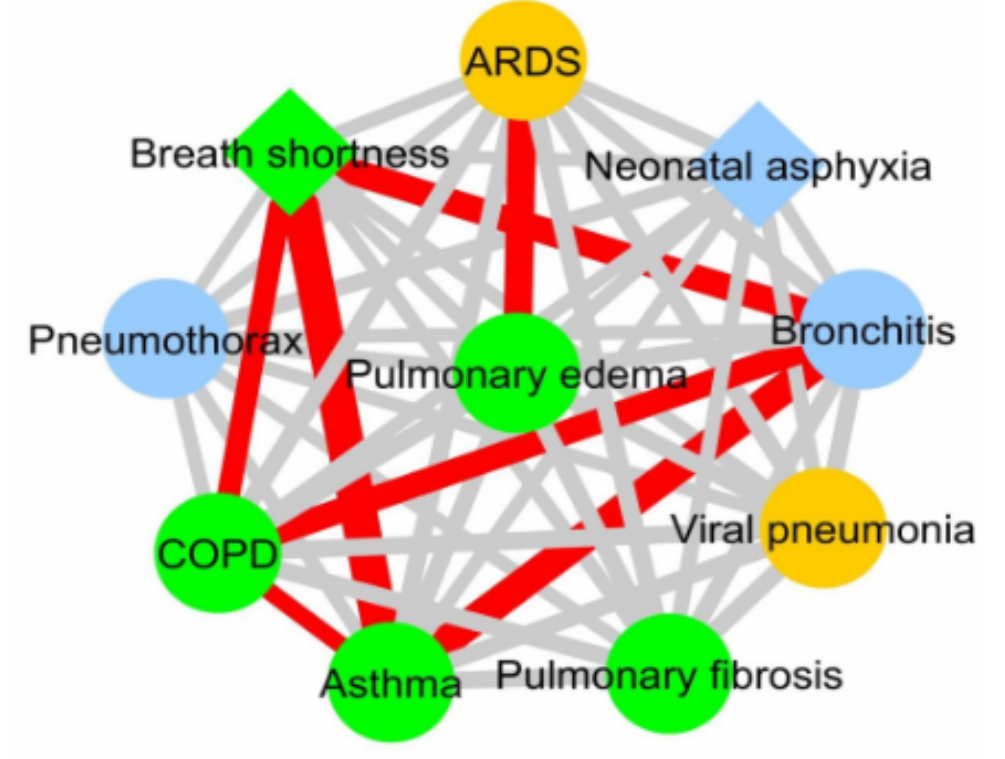

\section{Figure 3}

(A) The DO score based clustering of the 44 comorbid diseases of covid-19 were shown in dendrogram. The dendrogram showing five disease clusters namely clusters 1, 2, 3, 4 and 5. (B) In the Heatmap, scaled from 0 to 14, the rows are representing 35 diseases and the column are representing nine topological properties namely Neighborhood, Degree, Eccentricity, Average shortness, topological coefficient, Clustering coefficient, Closeness coefficient, radiality, betweenness of the each disease node. Please note 
that all the Cluster 1 (pulmonary related diseases) and their symptoms are ranked high in the network in terms of DO association score as well as the network properties (blue backgrounds). (C) Highly comorbid pulmonary diseases of COVID-19 represented in the subnetwork. The colors, shapes of the nodes, edges are as in Figure (2). The highly significant (DO score $>0.5$ ) edges are represented red lines

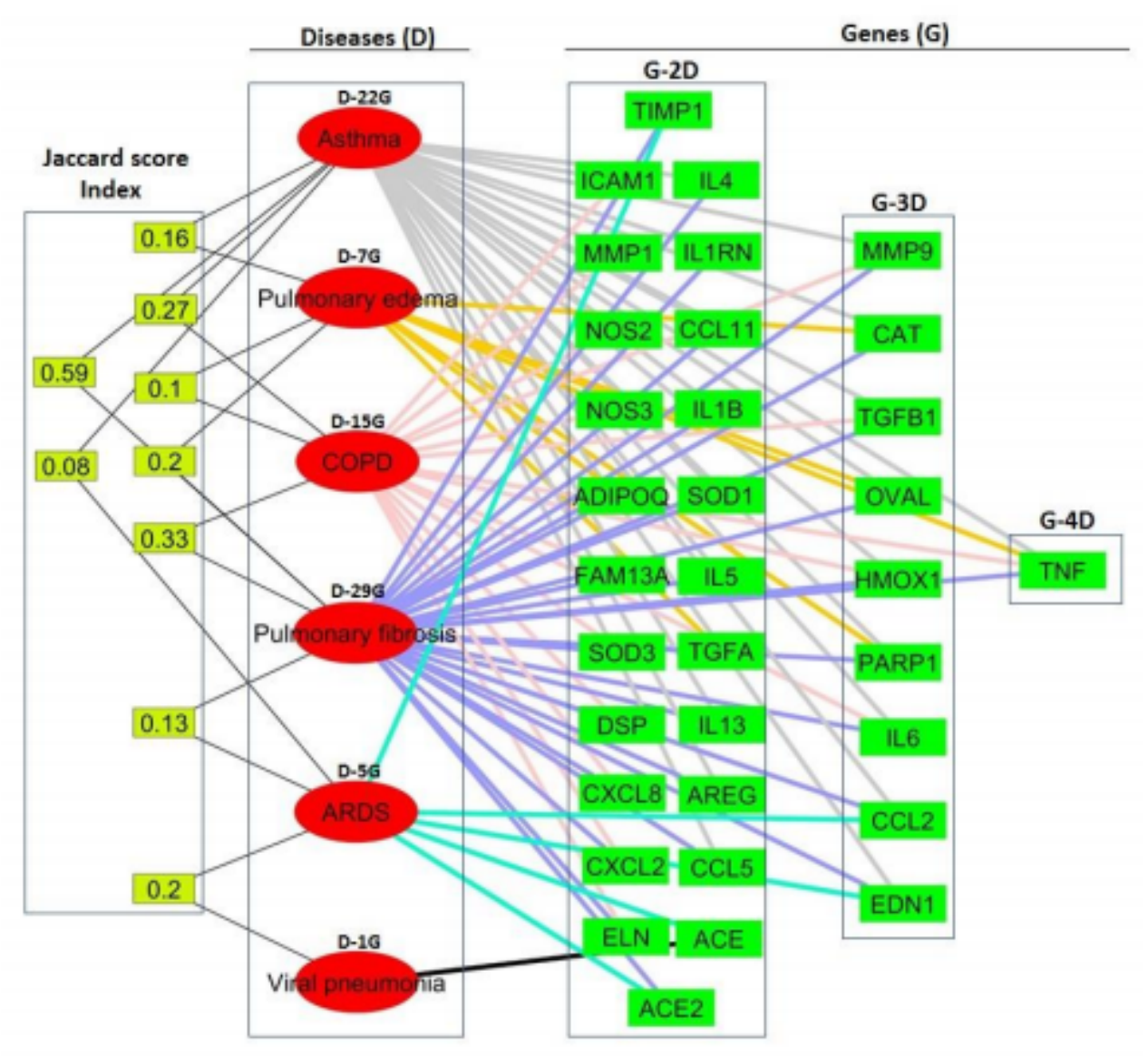

\section{Figure 4}

The strongly associated pulmonary diseases (D) in red ellipse (second row) and driver genes $(G)$ in green squares of row 3 (G-2D, genes having interaction more than 2 diseases), row 4 (G-3D, genes having interaction more than 3 diseases), row 5 (G-4D, genes having interaction more than 4 diseases), from Cluster 1 is shown as bipartite network. The interaction of each disease with its corresponding genes represented in different color (grey, yellow, violet, pink, blue and black). The number of disease-gene interactions are also mentioned say D-22G, represent the disease asthma interacting with 22 genes and so on. Similarly, the diseasegene-disease association represented in terms of Jaccard score (first row) and the values in square box. Please note the ARDS interacts with viral pneumonia and fibrosis through ACE and ACE2 respectively with the Jaccard score 0.2 . 


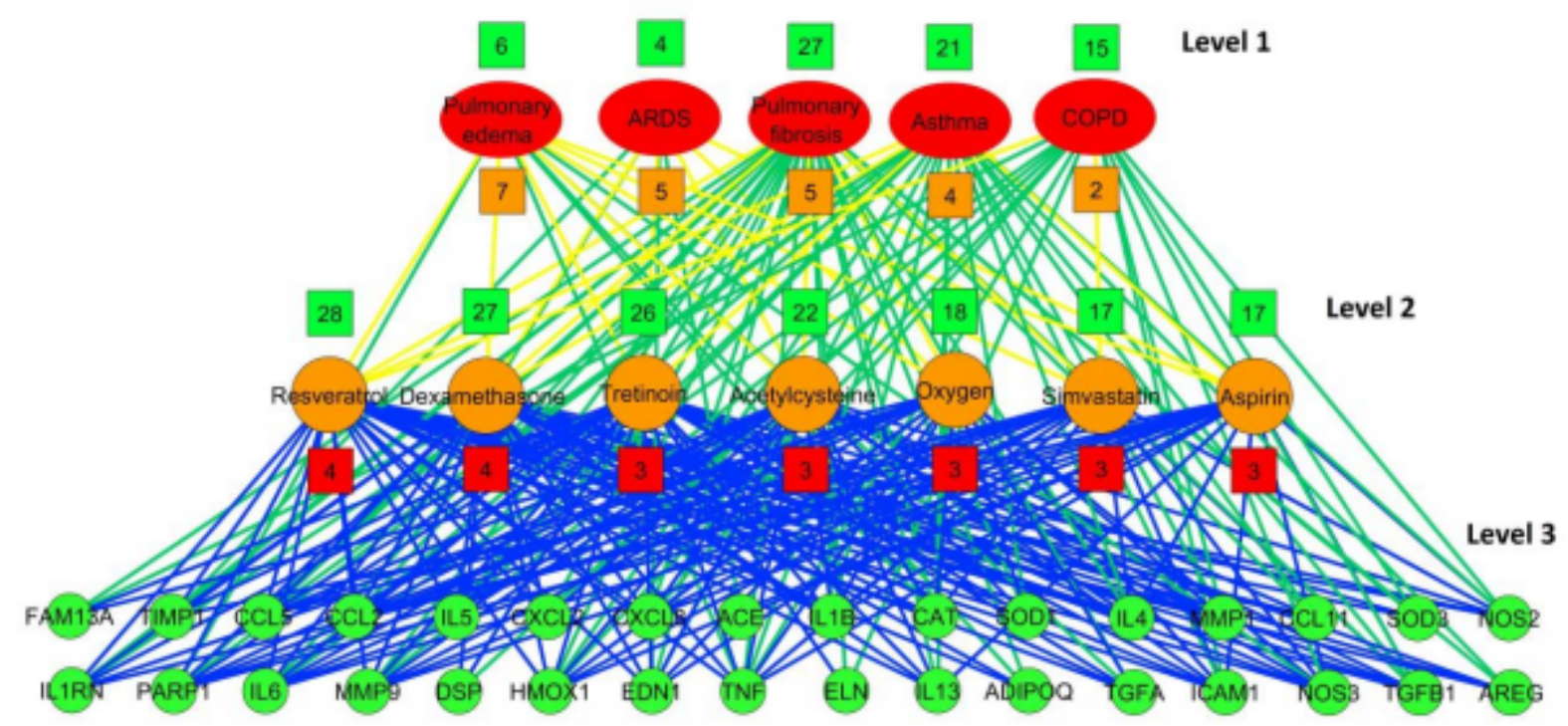

Figure 5

A tripartite network of pulmonary diseases node (red circles, level 1), drugs node (brown circles, level 2), genes node (green circles, level 3 ) is shown. The interaction between the different nodes are shown in three color lines. The edges of diseases with drugs and genes are yellow square and green squares respectively. The edges of drugs with diseases and genes are red and blue squares respectively. The numbers inside the squares above and below the circles represent the number of edges of the particular node. Please note that our predicated FDA approved drugs (6) and nutraceutical (1) are strongly interacting with driver genes as well as more than three pulmonary comorbid diseases of COVID-19. Notice the highest number of edges for the drug resveratrol in terms of diseases and genes. 


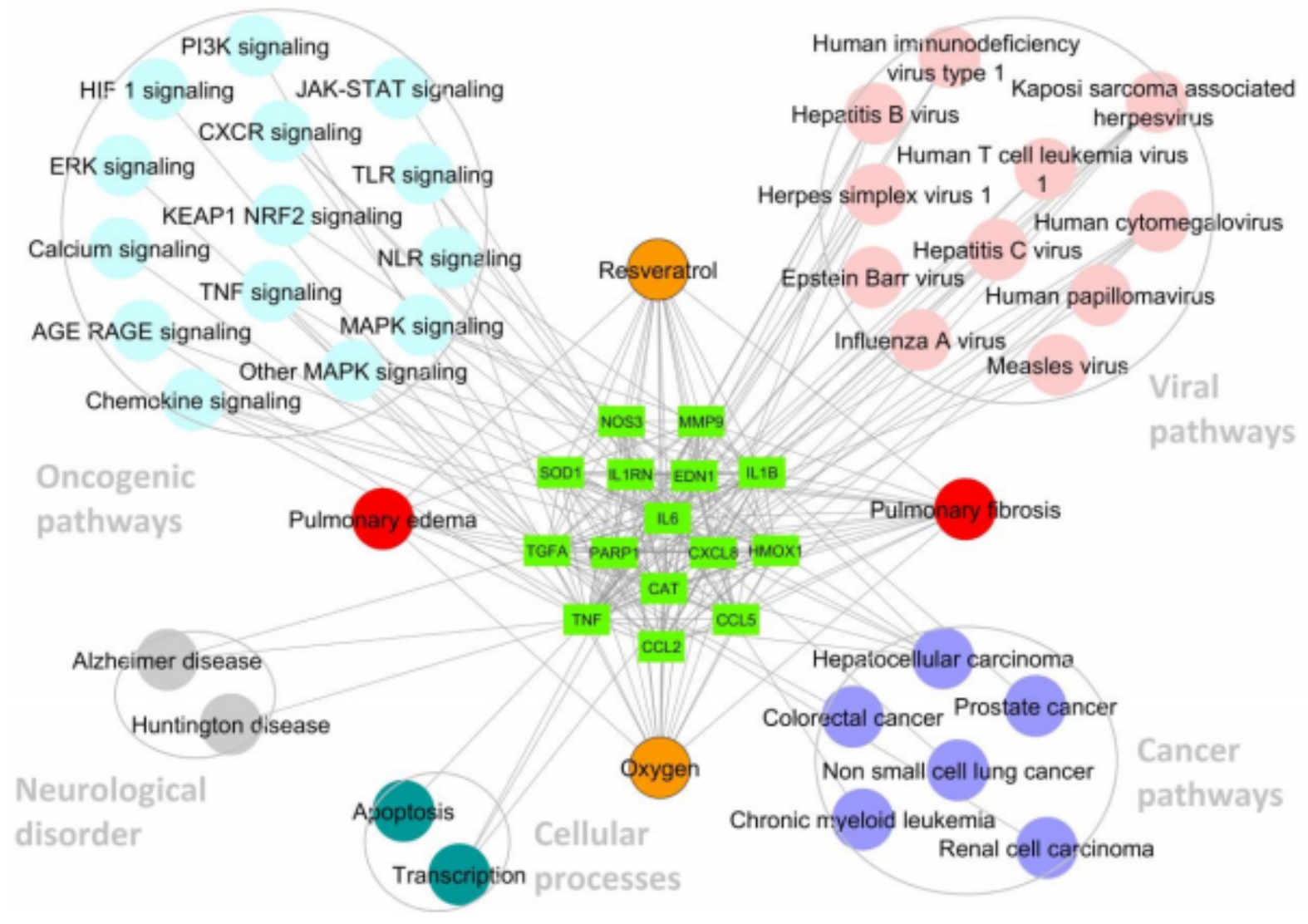

Figure 6

The resveratrol and oxygen chemicals (yellow circle) subgraph of tripartite network is shown (Figure 6). The 15 genes (out of 34 driver genes) highly perturbed by resveratrol drug are shown in green squares. Please note that the same 15 genes also shared by the chemical oxygen (yellow circle). These genes also perturbed during the disease manifestation of pulmonary fibrosis (red ellipse) and pulmonary edema (red ellipse). Notice that the functional enrichment of these genes strongly associate them with major oxygen signaling pathways (HIF, ERK, MAPK, TLR, .. in cyan circle) and leads to the exacerbation of several virus (pink circles) and cancers (violet circles). 


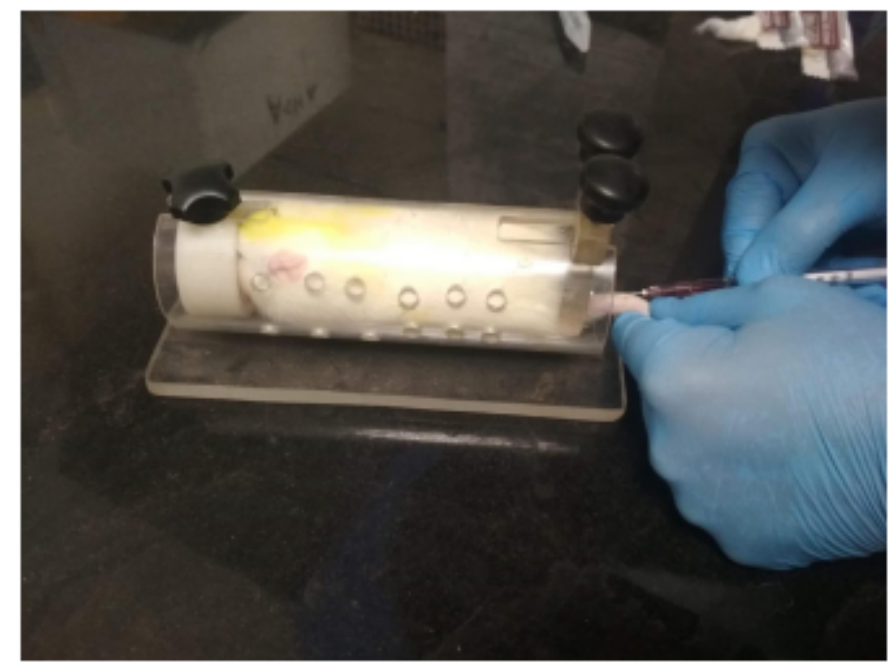

Figure 7A

Vascular leakage

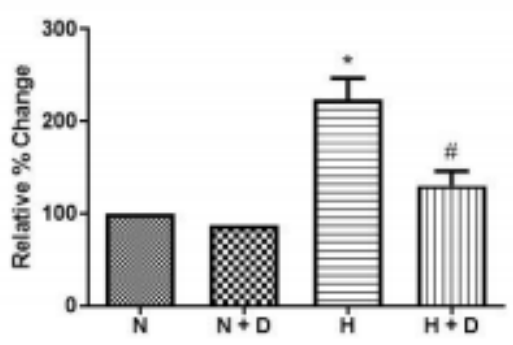

Figure $7 \mathrm{~B}$

\section{Figure 7}

(A) Rats were injected with drug in their tail vein. (B) Rats were exposed to simulated altitude of 25000 feet $(7620 \mathrm{~m})$ at $22^{\circ} \mathrm{C}$ for $8 \mathrm{~h}$ to observe the effect of Resveratrol $(15 \mathrm{mg} / \mathrm{kg} \mathrm{BW})$ on hypobaric hypoxiainduced vascular permeability. Values are mean $\pm S D(n=6)$. Significant test between groups were determined by using one-way ANOVA followed by Tukey test. * versus Control; \# versus Hypoxia. The experimental conditions were labelled as normoxia $(N)$, normoxia plus drug $(N+D)$, hypoxia $(H)$ and hypoxia plus drug $(H+D)$

\section{Supplementary Files}

This is a list of supplementary files associated with this preprint. Click to download.

- IdentificationofpulmonarycomorbiddiseasesnetworkbasedrepurposingeffectivedrugsforCOVID19.xlsx 